\title{
Anapole arising from a Mie scatterer with dipole excitation
}

\author{
Jorge R. Zurita-Sánchez (1) * \\ Instituto Nacional de Astrofísica, Óptica y Electrónica, Luis Enrique Erro 1, Tonantzintla, Puebla 72840, Mexico
}

(Received 27 June 2019; published 31 October 2019)

\begin{abstract}
We examine theoretically that an electric dipole close to a subwavelength sphere can yield an anapole (the dipole-sphere system is a nonradiating source). Physically, the anapole arises when the dipole induced by the source inside the sphere cancels out with the dipole contribution of the source with respect to the center of the sphere. The condition for the anapole is established and this state can be achieved with either a dielectric sphere with a high refractive index or a metallic sphere. Since there is a residual radiation (much smaller than the radiated power of the dipole in the absence of the scatterer) originating from high-order multipoles, the anapole is almost ideal. However, we show that the residual radiated power can be reduced even more by placing two electric dipoles on opposite sides of the spherical scatterer. This paper might have implications for controlling radiative properties of emitters and implementing applications related to molecular localization and sensing.
\end{abstract}

DOI: 10.1103/PhysRevResearch.1.033064

\section{INTRODUCTION}

The technological feasibility to grow and manipulate nanostructures and to tailor dielectric and magnetic properties with metamaterials has triggered unprecedentedly the examination of how electromagnetic energy is scattered in such objects. Examples of scattering effects include fluorescent enhancement [1], superscattering [2], highly directional antennas (originated from interference of several multipoles) [3-5], and particle localization [6].

In the context of Mie theory, an anapole can be considered as a nonradiating scatterer in which a nontrivial density current is induced. We can distinguish two physical mechanisms which give rise to an anapole. One of them requires multilayered particles in which the sum of induced dipoles on each layer vanishes, thus reduction of the scattering cross section is obtained [7,8]. In the other mechanism, the electromagnetic field distribution inside the particle corresponds to the superposition of Cartesian electric and toroidal dipoles which generate identical far-zone electromagnetic fields that interfere destructively [9]. Based on this destructive interference, anapoles generated from high-order multipoles (electric, magnetic, and toroidal) have been studied theoretically [10]. Furthermore, it is possible to create from a single multipolar current-charge distribution other high-order multipolar sources that are denominated moments of mean-square radii (MSRs) [11]; peculiarly, the radiation pattern of a MSR source is the same as that of its parent multipole and hence the superposition of these sources gives rise to an anapole.

\footnotetext{
*jrzurita@inaoep.mx

Published by the American Physical Society under the terms of the Creative Commons Attribution 4.0 International license. Further distribution of this work must maintain attribution to the author(s) and the published article's title, journal citation, and DOI.
}

In relation to the aforementioned former mechanism, we mention that those kinds of scatterers were not conceived as anapoles, but rather as invisibility cloaks. The first experimental observation of this cloaking effect was implemented with metamaterials for microwaves [12]. Also, it has been proposed that such effect can be applied for enhancing capabilities of near-field scanning optical microscopy $[13,14]$.

Concerning the mechanism of far-field destructive interference from electric and toroidal dipoles, the first experimental demonstration was achieved for microwave metamaterials [15]. Setups for realizing such anapoles require proper excitation schemes and particle geometric configurations to avoid radiation originating from unwanted high-order multipoles [16]. The pioneering experiment for observing an optical anapole used dielectric nanodisks excited by a plane wave [17]. Also under plane-wave illumination, it was proposed theoretically that plasmonic core-shell nanowires [18] and nanospheres [19] can yield anapoles, as well as spherical particles with anisotropic refractive index [20]. Moreover, as a consequence of anapole excitation, small scattering cross sections of subwavelength spheres with high refractive index [21] and metamolecules [22,23], and reduction of the scattered power by a planar optical metamaterial [24], can be attained. Also, high-order anapoles in a silicon disk excited by a plane wave have been examined and experimentally observed [25] and hybrid (a mixture of electric and magnetic responses) anapole modes in subwavelength spheres have been studied [26]. A dielectric sphere illuminated with two counterpropagating radially polarized beams can excite an ideal anapole mode [27], whereas illumination with azimuthally polarized beams can create a magnetic anapole [28]. In addition to cloaking applications of anapoles, they can be exploited for nanolasing [29] and enhancing nonlinear interactions [30-32].

The main goal of our paper is to show that the presence of a dipole in the vicinity of a sphere yields a different type of anapole which, in the lowest multipolar order, arises from the cancellation of the spherical dipole that is induced by the dipole source on the particle and the dipole contribution of the 
dipole source with respect to the center of the sphere. As will be seen, there is a small radiated power relative to the radiated power of the dipole source in the absence of the scatterer that arises from high-order multipoles. However, we will show that this residual emission can be reduced even more by placing two dipoles.

This paper is organized as follows. Section II presents the analytical expression for obtaining the electric field generated by a dipole with radial orientation in the vicinity of a sphere; from this expression, the radiation pattern and radiated power (far-field zone) are found, and importantly, the anapole condition is derived. Section III describes the results concerning the excitation of the anapole with a single electric dipole, while, in Sec. IV, the anapole generation arising from the presence of two dipoles is discussed. The last section is devoted to conclusions.

\section{THEORY}

A sphere has a radius $a$ and is centered at the origin; its dielectric (magnetic) function is $\epsilon_{2}\left(\mu_{2}\right)$. The background medium has dielectric and magnetic functions $\epsilon_{1}$ and $\mu_{1}$, respectively, and it is nonabsorbing $\left(\operatorname{Im}\left[\epsilon_{1}\right]=\operatorname{Im}\left[\mu_{1}\right]=0\right.$, where $\operatorname{Im}[\ldots]$ denotes imaginary part). We consider a point electric dipole that oscillates with angular frequency $\omega$ and has a dipole moment $\mathbf{p}$. We assume that the dipole is located outside the sphere and, without loss of generality, its position is $\mathbf{r}_{0}=z_{0} \mathbf{n}_{z}\left(z_{0}>a\right.$ and $\mathbf{n}_{z}$ is the unit Cartesian vector along the $z$ axis). Moreover, the dipole is oriented along the radial direction, that is, $\mathbf{p}=p_{0} \mathbf{n}_{z}$; a dipole with polar orientation cannot yield the effect that is treated in this paper.

\section{A. Electric field}

Outside the sphere, the electric field can be decomposed as

$$
\mathbf{E}_{\mathrm{T}}(\mathbf{r})=\mathbf{E}_{0}(\mathbf{r})+\mathbf{E}_{1}(\mathbf{r}),
$$

where $\mathbf{E}_{0}(\mathbf{r})$ is the direct field generated by the dipole as if the sphere was absent and $\mathbf{E}_{1}(\mathbf{r})$ is the electric field that is scattered by the sphere; we have omitted the time-dependent phasor $\exp (-i \omega t)$. Under the aforementioned conditions, the scattering field becomes

$$
\begin{aligned}
\mathbf{E}_{1}(\mathbf{r})= & \frac{i k_{1}^{3}}{4 \pi \varepsilon_{0} \epsilon_{1}} \sum_{l=1}^{\infty}(2 l+1) B_{l}(\omega) \frac{h_{l}\left(k_{1} z_{0}\right)}{k_{1} z_{0}} \\
& \times \overline{\mathbf{N}}_{e l 0}\left(\mathbf{r}, k_{1}\right) p_{0}
\end{aligned}
$$

where $\varepsilon_{0}$ is the vacuum permittivity, $k_{1}=\sqrt{\epsilon_{1} \mu_{1}} k_{0}\left(k_{0}=\right.$ $\omega / c$, where $c$ is the light speed in vacuum) is the wave vector in the background medium, and $h_{l}(u)$ is the spherical Hankel function of first kind of order $l$. The vector spherical harmonic is

$$
\overline{\mathbf{N}}_{\sigma l m}(\mathbf{r}, k)=\frac{1}{k} \nabla \times\left\{\nabla \times\left[\mathbf{r} h_{l}(k r) y_{\sigma l m}(\theta, \phi)\right]\right\},
$$

where $(r, \theta, \phi)$ are the spherical coordinates of $\mathbf{r}, \sigma=$ $e$ and $o, y_{\text {elm }}(\theta, \phi) \equiv P_{l}^{m}(\cos \theta) \cos (m \phi)$, and $y_{o l m}(\theta, \phi) \equiv$ $P_{l}^{m}(\cos \theta) \sin (m \phi) \quad\left[P_{l}^{m}(\cos \theta)\right.$ is the associated Legendre function of order $m$ and degree $l$ which is explicitly defined as $P_{l}^{m}(u) \equiv\left(1-u^{2}\right)^{m / 2}\left(d^{m} / d u^{m}\right) P_{l}(u)$ where $P_{l}(u)$ is the ordinary Legendre polynomial of order $l$ ]. The TM Mie coefficient is

$$
B_{l}(\omega)=\frac{\epsilon_{2} j_{l}\left(\rho_{2}\right) \psi_{l}^{\prime}\left(\rho_{1}\right)-\epsilon_{1} j_{l}\left(\rho_{1}\right) \psi_{l}^{\prime}\left(\rho_{2}\right)}{\epsilon_{1} h_{l}\left(\rho_{1}\right) \psi_{l}^{\prime}\left(\rho_{2}\right)-\epsilon_{2} j_{l}\left(\rho_{2}\right) \xi_{l}^{\prime}\left(\rho_{1}\right)},
$$

where $\rho_{i}=k_{i} a$ ( $i=1$ and 2 and $\left.k_{2}=\sqrt{\epsilon_{2} \mu_{2}} k_{0}\right), \quad \xi_{l}(u) \equiv$ $u h_{l}(u)$ is the Riccati-Hankel function of order $l$, and $\psi_{l}(u) \equiv$ $u j_{l}(u)$ is the Riccati-Bessel function of order $l\left[j_{l}(u)\right.$ is the spherical Bessel function of first kind of order $l]$. We state that a primed function means the derivative with respect to its argument. We mention that (2) follows from the expansion of the Green tensor in terms of vector spherical harmonics [33] and the fact that

$$
\overline{\mathbf{N}}_{\sigma l m}\left(z_{0} \mathbf{n}_{z}, k\right) \cdot \mathbf{n}_{z}=\delta_{0 m} \delta_{e \sigma} l(l+1) h_{l}\left(k z_{0}\right) /\left(k z_{0}\right),
$$

where $\delta_{i j}$ is the Kronecker- $\delta$ tensor.

The direct field can be also expressed in a vector spherical harmonics series with respect to the center of the sphere as [34]

$$
\mathbf{E}_{0}(\mathbf{r})=\frac{i k_{1}^{3}}{4 \pi \varepsilon_{0} \epsilon_{1}} \sum_{l=1}^{\infty}(2 l+1)\left\{\begin{array}{ll}
{\left[h_{l}\left(k_{1} z_{0}\right) /\left(k_{1} z_{0}\right)\right] \mathbf{N}_{e l 0}\left(\mathbf{r}, k_{1}\right) p_{0},} & |\mathbf{r}|<z_{0} \\
{\left[j_{l}\left(k_{1} z_{0}\right) /\left(k_{1} z_{0}\right)\right] \overline{\mathbf{N}}_{e l 0}\left(\mathbf{r}, k_{1}\right) p_{0},} & |\mathbf{r}|>z_{0}
\end{array},\right.
$$

where the spherical harmonic $\mathbf{N}_{\sigma l m}(\mathbf{r}, k)$ is defined as (3), but $h_{l}(k r)$ is replaced by $j_{l}(k r)$. Again, the simplified expression (6) is a consequence of (5), and the fact that $\mathbf{N}_{\sigma l m}\left(z_{0} \mathbf{n}_{z}, k\right) \cdot \mathbf{n}_{z}$ is the same as (5), but $h_{l}(u) \rightarrow j_{l}(u)$.

For completeness, the electric field inside the sphere is

$$
\mathbf{E}_{2}(\mathbf{r})=\frac{i k_{1}^{3}}{4 \pi \varepsilon_{0} \epsilon_{1}} \sum_{l=1}^{\infty}(2 l+1) D_{l}(\omega) \frac{h_{l}\left(k_{1} z_{0}\right)}{k_{1} z_{0}} \mathbf{N}_{e l 0}\left(\mathbf{r}, k_{2}\right) p_{0} .
$$

Here, the transmission Mie coefficient of TM waves is

$$
D_{l}(\omega)=\frac{-i \epsilon_{2}}{\rho_{1}\left[\epsilon_{1} h_{l}\left(\rho_{1}\right) \psi_{l}^{\prime}\left(\rho_{2}\right)-\epsilon_{2} j_{l}\left(\rho_{2}\right) \xi_{l}^{\prime}\left(\rho_{1}\right)\right]} \frac{Z_{2}}{Z_{1}} .
$$

Here, $Z_{i}=\left[\left(\mu_{0} \mu_{i}\right) /\left(\varepsilon_{0} \epsilon_{i}\right)\right]^{1 / 2}\left(i=1\right.$ and 2 , and $\mu_{0}$ is the vacuum permeability) is the impedance of the corresponding medium.

\section{B. Far-field limit}

To calculate the far-field limit of the scattering field $\left(k_{1} r \rightarrow\right.$ $\infty)$, we use the asymptotic limit for the spherical Hankel function:

$$
\lim _{\rho \rightarrow \infty} h_{l}(\rho)=e^{i \rho} /\left(i^{l+1} \rho\right) .
$$


By using (9) in Eqs. (2) and (6), the total far-field electric field becomes

$$
\begin{aligned}
\mathbf{E}_{\mathrm{T} \infty}(\mathbf{r})= & \frac{k_{1}^{2}}{4 \pi \varepsilon_{0} \epsilon_{1}} \frac{i e^{i k_{1} r}}{r} \sum_{l=1}^{\infty} \frac{(2 l+1)}{i^{l} k_{1} z_{0}}\left[j_{l}\left(k_{1} z_{0}\right)\right. \\
& \left.+B_{l}(\omega) h_{l}\left(k_{1} z_{0}\right)\right] \mathbf{Q}_{e l 0}(\theta, \phi) p_{0} .
\end{aligned}
$$

Here,

$$
\begin{aligned}
\mathbf{Q}_{e_{l}}(\theta, \phi)= & \frac{d}{d \theta} P_{l}^{m}(\cos \theta)_{\sin (m \phi)}^{\cos (m \phi)} \mathbf{n}_{\theta} \\
& \mp \frac{P_{l}^{m}(\cos \theta)}{\sin \theta} m_{\cos (m \phi)}^{\sin (m \phi)} \mathbf{n}_{\phi},
\end{aligned}
$$

where $\mathbf{n}_{\theta}$ and $\mathbf{n}_{\phi}$ are the polar and azimuthal unit vectors, respectively. From the Faraday-Maxwell equation, the total magnetic field in the radiation zone is

$$
\begin{aligned}
\mathbf{H}_{\mathrm{T} \infty}(\mathbf{r})= & \frac{k_{1}^{2}}{4 \pi \varepsilon_{0} \epsilon_{1} Z_{1}} \frac{e^{i k_{1} r}}{r} \sum_{l=1}^{\infty} \frac{(2 l+1)}{i^{l+1} k_{1} z_{0}}\left[j_{l}\left(k_{1} z_{0}\right)\right. \\
& \left.+B_{l}(\omega) h_{l}\left(k_{1} z_{0}\right)\right]\left[\mathbf{Q}_{e l 0}(\theta, \phi) \times \mathbf{n}_{r}\right] p_{0},
\end{aligned}
$$

where $\mathbf{n}_{r}$ is the radial unit vector.

\section{Radiated power}

The time-average far-field Poynting vector is

$$
\langle\mathbf{S}(\mathbf{r}, t)\rangle=\frac{1}{2} \operatorname{Re}\left[\mathbf{E}_{\mathrm{T} \infty}^{*}(\mathbf{r}) \times \mathbf{H}_{\mathrm{T} \infty}(\mathbf{r})\right],
$$

where $\operatorname{Re}[\ldots]$ denotes the real part. Then, the radiated power is the amount of energy that goes through a spherical surface with large radius $R$, that is,

$$
P=\int_{A} \frac{d P(\theta, \phi)}{d \Omega} d \Omega,
$$

where $d \Omega$ is the solid angle differential and

$$
\frac{d P(\theta, \phi)}{d \Omega}=R^{2}\langle\mathbf{S}(R, \theta, \phi ; t)\rangle \cdot \mathbf{n}_{r}
$$

is the so-called radiation pattern. By using (10) and (12), the radiation pattern (15) and the radiated power (14) become

$$
\begin{aligned}
\frac{1}{P_{0}} \frac{d P(\theta, \phi)}{d \Omega}= & \frac{3}{8 \pi} \operatorname{Re}\left[\sum_{l, l^{\prime}=1}^{\infty} \frac{(2 l+1)}{(-i)^{l}} \frac{\left(2 l^{\prime}+1\right)}{i^{l^{\prime}}} \gamma_{l}^{*}\left(k_{1} z_{0}\right)\right. \\
& \left.\times \gamma_{l^{\prime}}\left(k_{1} z_{0}\right) \frac{d}{d \theta} P_{l}^{0}(\cos \theta) \frac{d}{d \theta} P_{l^{\prime}}^{0}(\cos \theta)\right]
\end{aligned}
$$

and

$$
\frac{P}{P_{0}}=\frac{3}{2} \sum_{l=1}^{\infty} l(l+1)(2 l+1)\left|\gamma_{l}\left(k_{1} z_{0}\right)\right|^{2},
$$

respectively. Here

$$
\gamma_{l}(\rho)=\left[j_{l}(\rho)+B_{l}(\omega) h_{l}(\rho)\right] / \rho,
$$

and $P_{0}$ is the radiated power by a dipole when the sphere is absent, which is given by

$$
P_{0}=\frac{1}{3} \frac{\omega k_{1}^{3}\left|p_{0}\right|^{2}}{4 \pi \varepsilon_{0} \epsilon_{1}} .
$$

Equation (17) follows from orthogonality properties of spherical harmonics [35].

\section{Condition for the anapole}

According to (10), the radiation of the $l$ th order can be suppressed if

$$
j_{l}\left(k_{1} z_{0}\right)+B_{l}(\omega) h_{l}\left(k_{1} z_{0}\right)=0 .
$$

As mentioned, physically, the induced $l$ th-order multipole on the particle by the source is the same as the $l$ th multipolar contribution of the exciting dipole (in the absence of the scatterer) with respect to the center of the particle, but the phase difference between such multipolar distributions is $\pi \mathrm{rad}$.

As we will see, for particles with subwavelength size, the contribution of the lowest multipolar order (dipole) accounts almost for the total of the radiated power. Consequently, if (20) is satisfied for $l=1$, the radiated power for a subwavelength sphere is practically null. Hence the dipole and scatterer constitute an anapole.

\section{RESULTS}

We assume that the scatterer is nonmagnetic $\left(\mu_{2}=1\right)$ and it is embedded in vacuum $\left(\epsilon_{1}=\mu_{1}=1\right.$, thus $\left.k_{1}=k_{0}\right)$. For a dipole close to a subwavelength sphere, the condition (20) for $l=1$ can be fulfilled when the particle has a high refractive index $\left(\sqrt{\epsilon_{2}}\right)$ or a negative dielectric function (metallic character). Next we discuss these cases.

\section{A. Dielectric sphere}

The dielectric function $\epsilon_{2}$ that fulfills the anapole condition versus the surface-dipole separation $k_{0}(z-a)$ for several subwavelength particle sizes is shown in Fig. 1(a). As seen in the curve, to fulfill the anapole condition, the sphere must have a high refractive index. Also we can notice that the dielectric function $\epsilon_{2}$ is reduced noticeably (by about a factor of 4) as the size of the particle $k_{0} a$ increases from 0.2 to 0.4. However, for a fixed value of size $k_{0} a$, the dielectric function decreases slowly as the dipole-surface separation $k_{0}\left(z_{0}-a\right)$ increases. Unfortunately the refractive index of natural dielectric materials for optical frequencies cannot reach the refractive index associated to the dielectric functions of Fig. 1(a). However, there are metamaterials with a high refractive index for terahertz waves [36], allowing the possible experimental realization of this anapole.

Figure 1(b) depicts the normalized radiated power with respect to the radiated power in free space $\left(P / P_{0}\right)$ as a function of the surface-dipole separation for several sphere sizes. Of course, the multipolar contribution of the lowest order $(l=1)$ to $P / P_{0}$ vanishes, thus high-order multipoles impact the radiated power. More precisely, for the sizes and surface-dipole distances of Fig. 1(b), the quadrupole $(l=2)$ accounts for at least $98.6 \%$ of the total radiated power. We can notice that, for subwavelength particles, the radiated power is reduced with respect to $P_{0} ; P / P_{0}$ falls as low as 0.025 for $k_{0} a=0.2$. Moreover, as seen in Fig. 1(b), $P / P_{0}$ increases as the size $k_{0} a$ increases and it augments appreciably as the dipole becomes close to the surface. 
(a)

$\lambda_{0}=2 \pi \times 100 \mathrm{~nm}$

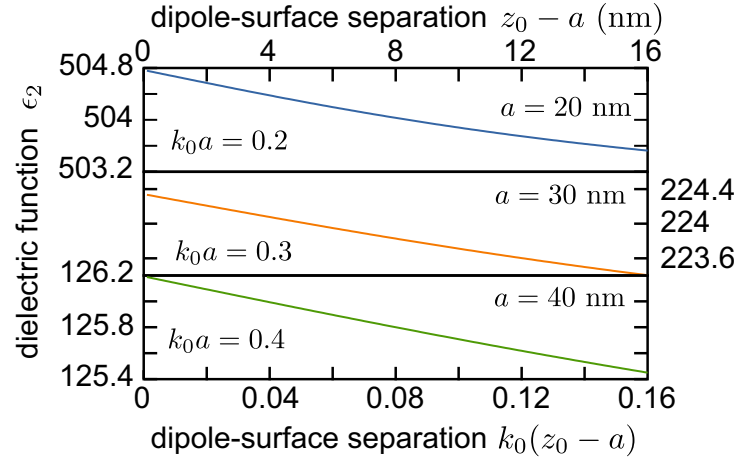

(b)

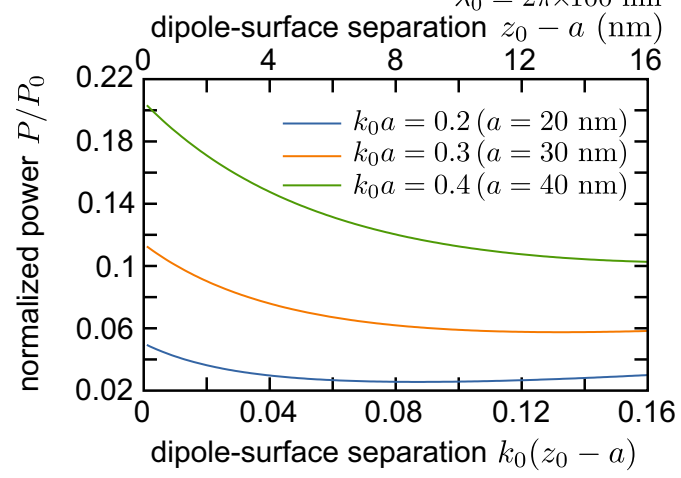

(c)

$\lambda_{0}=2 \pi \times 100 \mathrm{~nm}$

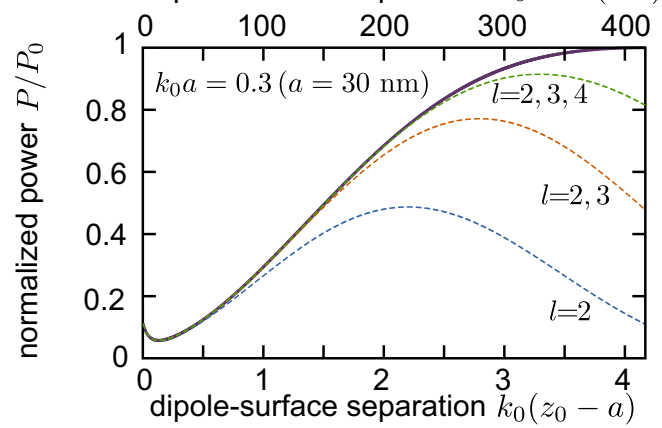

FIG. 1. Dielectric sphere. (a) The dielectric function $\epsilon_{2}$ vs the dipole-surface separation $k_{0}\left(z_{0}-a\right)$ for which condition (20) is fulfilled for $l=1$ and $k_{0} a=0.2,0.3$, and 0.4. (b) The normalized power $P / P_{0}$ against dipole-surface separation $k_{0}\left(z_{0}-a\right)$ for $k_{0} a=0.2,0.3$, and 0.4 . (c) The normalized power $P / P_{0}$ vs large dipole-surface separations for $k_{0} a=0.3$, including partial multipolar contributions ( $l=2 ; 2$ and 3 ; and 2-4). Conversion to nanometers is included for an anapole occurring at a free-space wavelength $\lambda_{0}=2 \pi \times 100 \mathrm{~nm}$.

Figure 1(c) for particle size $k_{0} a=0.3$ shows how the normalized radiated power varies for larger dipole-surface separations when the anapole condition (20) for $l=1$ is satisfied. We can notice that the normalized radiated power increases monotonically from $k a \approx 0.13$ and it reaches almost the free-space value $\left(P / P_{0}=1\right)$ when $k_{0}\left(z_{0}-a\right) \gtrsim 4$. Hence, the condition for low radiated power can be attained when the dipole is nearby the sphere. Also we can appreciate that the quadrupolar contribution $l=2$ of $P / P_{0}$ is dominant for a small dipole-surface distance $\left(k_{0}\left(z_{0}-a\right) \lesssim 0.6\right)$. As (a)

$$
\log _{2}\left[a^{3} \varepsilon_{0}\left|\mathbf{E}(\mathbf{r}, 0) / p_{0}\right|\right] \quad l=1
$$
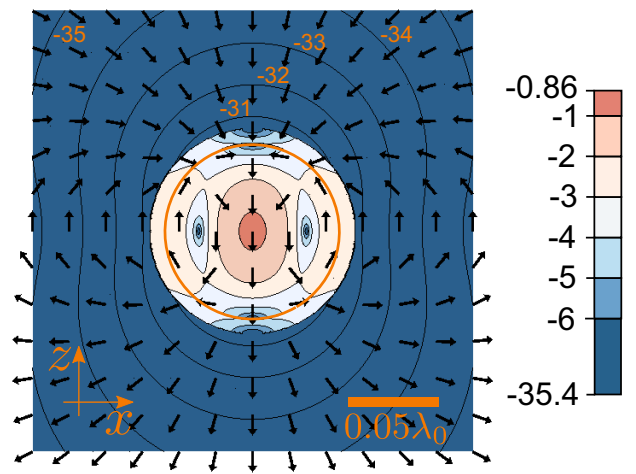

(b)

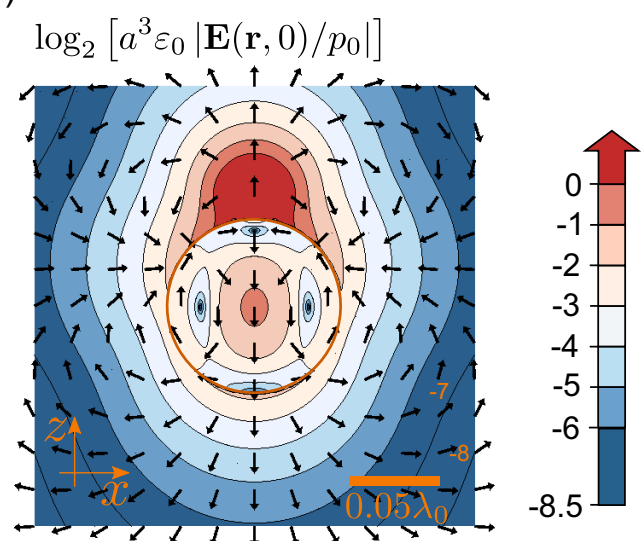

(c)
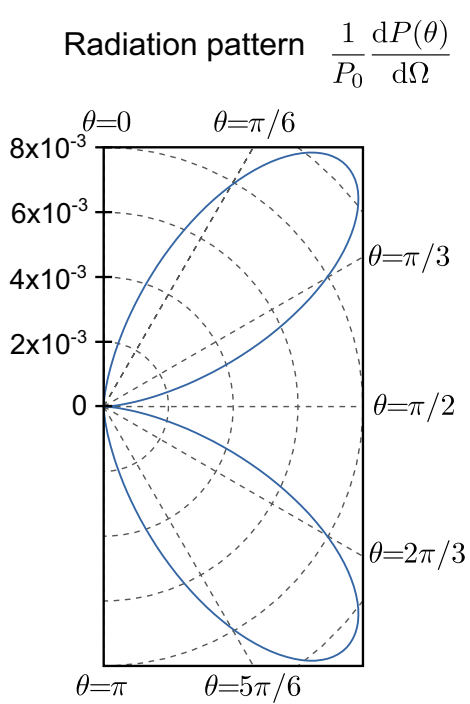

FIG. 2. Dielectric sphere. Here, $k_{0} a=0.3$ and $k_{0}\left(z_{0}-a\right)=$ 0.05. (a) Contour plot of the dipolar contribution $(l=1)$ of $\left.\log _{2}\left[a^{3} \varepsilon_{0} \mid \mathbf{E}(\mathbf{r}, 0)\right] / p_{0} \mid\right]$ in the $x z$ plane. (b) Contour plot of $\log _{2}\left[a^{3} \varepsilon_{0}\left|\mathbf{E}(\mathbf{r}, 0) / p_{0}\right|\right]$ in the $x z$ plane. (c) Radiation pattern $(d P / d \Omega) / P_{0}$ vs $\theta$.

$k_{0}\left(z_{0}-a\right)$ grows, high-order multipoles contribute to the radiated power.

Now we consider the case in which $k_{0} a=0.3$ and $k_{0}\left(z_{0}-\right.$ $a)=0.05$. For this size and surface dipole, the anapole 


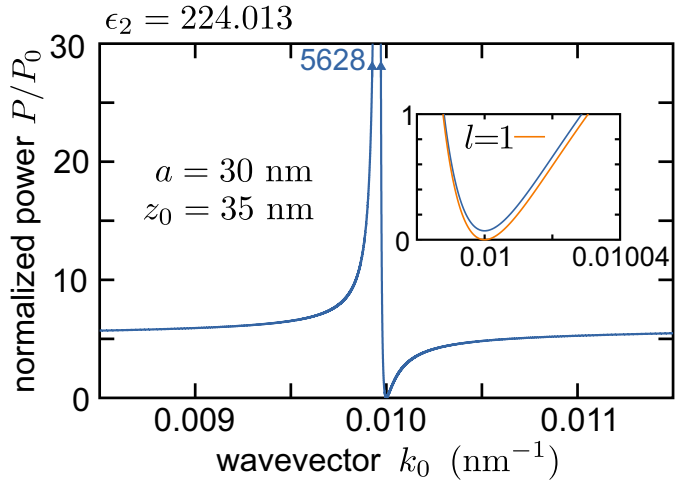

FIG. 3. Normalized power $P / P_{0}$ vs the free-space wave vector $k_{0}$ for $a=30 \mathrm{~nm}$ and $z_{0}=35 \mathrm{~nm}$. The dielectric constant of the sphere is $\epsilon_{2}=224.013$. The inset shows an enlargement around the the frequency where the anapole happens, including the dipole contribution $(l=1)$ of $P / P_{0}$

condition is matched when $\epsilon_{2}=224.013$ and this setup yields $P / P_{0}=0.071$. Now we define

$$
\mathbf{E}(\mathbf{r}, t)= \begin{cases}\operatorname{Re}\left[\mathbf{E}_{\mathrm{T}}(\mathbf{r}) e^{-i \omega t}\right], & |\mathbf{r}|>a \\ \operatorname{Re}\left[\mathbf{E}_{2}(\mathbf{r}) e^{-i \omega t}\right], & |\mathbf{r}|<a\end{cases}
$$

In Fig. 2(a), we depict the contour plot ( $x z$ plane) of the dipole contribution $(l=1)$ of the magnitude of the electric field $\mathbf{E}(\mathbf{r}, 0)$ as well as its direction. This distribution corresponds to the anapole where it can be noticed that the dipole contribution of $|\mathbf{E}(\mathbf{r}, 0)|$ in the region beyond the source is at least about nine orders of magnitude smaller than that in the center of the sphere. We mention that there is a discontinuity of the dipole contribution of the electric field at surface $r=z_{0}$ which arises from the different vector spherical harmonic bases in which the direct field $\mathbf{E}_{0}(\mathbf{r})$ is expanded [see (6)]. The total magnitude of the electric field is depicted in Fig. 2(b), where it can be observed that, in the vicinity of the particle, the high-order multipolar contributions of the electric field are significant. However, as mentioned, the residual radiated power arises from the induced quadrupole in the sphere. As observed in Fig. 2(c), the radiation pattern is indeed originated from a quadrupole source.

Next we describe the spectral dependence of the radiated power around the anapole condition which is satisfied assumedly when $k_{0}=0.01 \mathrm{~nm}^{-1}, a=30 \mathrm{~nm}, z_{0}=35 \mathrm{~nm}$, and $\epsilon_{2}=224.013$. Furthermore we consider that $\epsilon_{2}$ is spectrally constant. Figure 3 depicts the normalized power $P / P_{0}$ as a function of the free-space wave vector $k_{0}$. We can notice that the spectral region of low radiated power, where the anapole occurs, is preceded immediately by a resonance of a whispering gallery mode associated to the dipole $(l=1)$. The inset of Fig. 3 shows that the dipole contribution $(l=1)$ of $P / P_{0}$ vanishes when the anapole condition is fulfilled.

\section{B. Metallic sphere}

As stated, the particle's dielectric function $\epsilon_{2}$ satisfying condition (20) for $l=1$ can exhibit metallic character. This is illustrated in the curve $\epsilon_{2}$ against $k_{0}\left(z_{0}-a\right)$ of Fig. 4(a). As seen the dielectric constant is negative and near zero when surface-dipole separation becomes small $\left[k_{0}\left(z_{0}-a\right) \ll 1\right]$; (a)

$$
\lambda_{0}=2 \pi \times 100 \mathrm{~nm}
$$

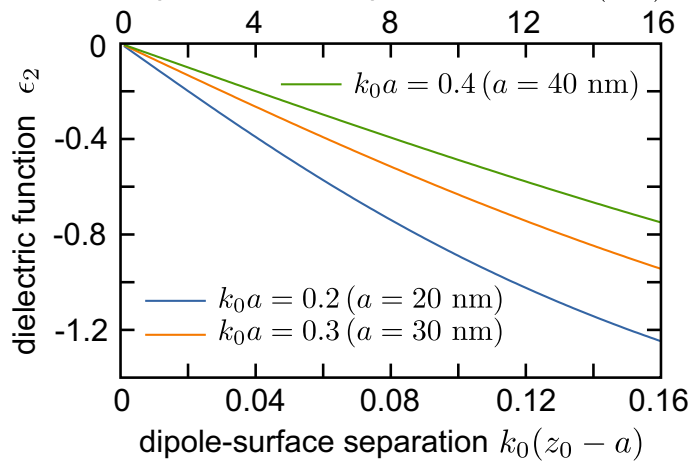

(b)

$$
\lambda_{0}=2 \pi \times 100 \mathrm{~nm}
$$

dipole-surface separation $z_{0}-a(\mathrm{~nm})$

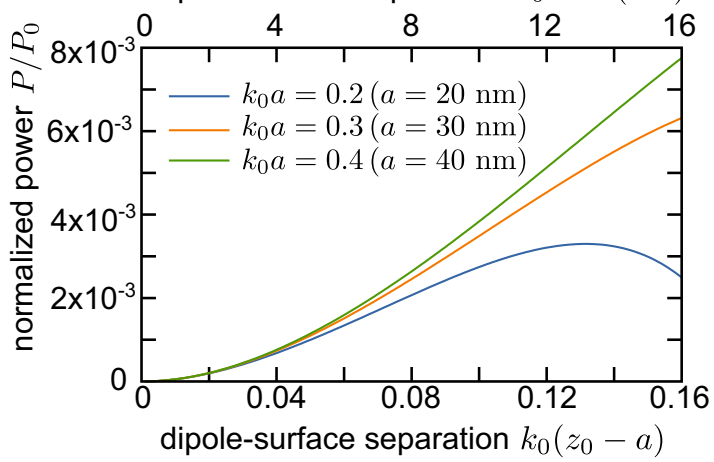

(c)

$$
\lambda_{0}=2 \pi \times 100 \mathrm{~nm}
$$
dipole-surface separation $z_{0}-a(\mathrm{~nm})$

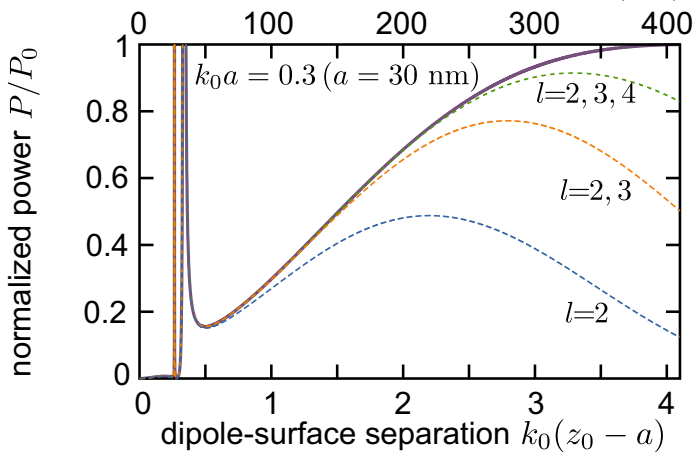

FIG. 4. Metallic sphere. The dielectric function $\epsilon_{2}$ vs the dipolesurface separation $k_{0}\left(z_{0}-a\right)$ for which condition (20) is fulfilled for $l=1$ and $k_{0} a=0.2,0.3$, and 0.4. (b) The normalized power $P / P_{0}$ against dipole-surface separation $k_{0}\left(z_{0}-a\right)$ for $k_{0} a=0.2,0.3$, and 0.4. (c) The normalized power $P / P_{0}$ vs large dipole-surface separations for $k_{0} a=0.3$, including partial multipolar contributions ( $l=2 ; 2$ and 3; and 2-4). Conversion to nanometers is included for an anapole occurring at a free-space wavelength $\lambda_{0}=2 \pi \times 100 \mathrm{~nm}$.

then $\epsilon_{2}$ decreases as $k_{0}\left(z_{0}-a\right)$ increases. The larger the particle size is, the smaller $\epsilon_{2}$ becomes. Figure 4(b) plots the radiated power as a function of the surface-dipole distance $k_{0}\left(z_{0}-a\right)$ for the anapole. Contrary to the case of the sphere with high refractive index, the normalized radiated power $P / P_{0}$ practically vanishes as $k_{0}\left(z_{0}-a\right) \rightarrow 0$. In addition, $P / P_{0}$ increases as $k_{0}\left(z_{0}-a\right)$ increases, except for the particle size $k_{0} a=0.2$ in which the curve goes downwards from 
$k\left(z_{0}-a\right)=0.13$. For fixed values of $a$ and $z_{0}$, relative to the sphere with high refractive index, the normalized radiated power $P / P_{0}$ is even smaller. Also, for the interval of $k_{0}\left(z_{0}-a\right)$ and sizes of Fig. 4(a), the quadrupole part of the radiated power is as low as $94.6 \%$.

Again, we consider a sphere with size $k_{0} a=0.3$. The plot of the normalized radiated power for large dipole-surface distances is illustrated in Fig. 4(c) in which the anapole condition (20) for $l=1$ is fulfilled. For $k_{0}\left(z_{0}-a\right)>0.5$, the curve $P / P_{0}$ exhibits the same behavior as that of the dielectric sphere [see Fig. 1(c)], namely, it increases as the dipole-surface distance increases until $k_{0}\left(z_{0}-a\right) \sim 4$ where $P / P_{0} \rightarrow 1$ (free-space limit). In the interval $k_{0}\left(z_{0}-a\right)<0.5$, we can notice the aforementioned region of low-radiated power which is followed by two peaks associated to plasmonic resonances; the first (second) peak is due to order $l=3(l=2)$ where $\epsilon_{2}$ is close to $-1.33(-1.5)$. Like the dielectric sphere case, as $k_{0}\left(z_{0}-a\right)$ increases, more high-order multipoles contribute to the radiated power.

For comparison purposes, we consider a metallic sphere with same radius $k_{0} a=0.3$ and surface-source separation $k_{0}\left(z_{0}-a\right)=0.05$ as the case of the particle with high refractive index. The dielectric constant satisfying the anapole condition is $\epsilon_{2}=-0.329$. The normalized radiated power is $P / P_{0}=1.098 \times 10^{-3}$, which is 1.8 orders of magnitude smaller than the case of the dielectric sphere. The contour plot ( $x z$ plane) of the magnitude of dipole contribution (anapole) of the electric field $\mathbf{E}(\mathbf{r}, 0)$ is shown in Fig. 5(a). Again, as a characteristic feature of the anapole, we can appreciate that this partial distribution of the magnitude of the electric field $|\mathbf{E}(\mathbf{r}, 0)|$ in the external region $\left(k_{0} r>0.35\right)$ is considerably smaller (about 13 orders of magnitude) than that in the internal region $\left(k_{0} r<0.35\right)$. Contrary to the dielectric sphere case, the dipole contribution of the electric field inside the sphere is uniform and polarized in the $z$ direction. Figure 5(b) depicts the total magnitude of the electric field $|\mathbf{E}(\mathbf{r}, 0)|$ where it shows that high-order multipolar orders are responsible for the near field (inside and nearby the sphere). Also, as shown in Fig. 5(c), the low-intensity radiation pattern arises from the induced quadrupole on the sphere.

To study the spectral response of the radiated power for a metallic sphere, we consider that the dipole position is $z_{0}=35 \mathrm{~nm}$, the sphere radius is $a=30 \mathrm{~nm}$, and the dielectric function of the metal is described by the plasma model, namely, $\epsilon_{2}\left(k_{0}\right)=1-k_{\mathrm{p}}^{2} / k_{0}^{2}$ ( $k_{\mathrm{p}}$ is the plasma frequency). Furthermore, we assume that the anapole condition is satisfied when $k_{0}=0.01 \mathrm{~nm}^{-1}$, which yields $\epsilon_{2}=-0.329$. Consequently, we have chosen a hypothetical metal with plasma frequency $k_{\mathrm{p}}$ such that $\epsilon_{2}\left(k_{0}=0.01 \mathrm{~nm}^{-1}\right)=-0.329$. Under the aforementioned conditions, Fig. 6 shows the normalized radiated power $P / P_{0}$ as a function of the free-space wave vector. As seen, differently from the dielectric response, the $P / P_{0}$ curve for the metallic sphere has a broad spectral dip and the minimum of which happens at the anapole frequency; the inset of Fig. 6 zooms in on the region where the radiated power is minimum and it shows that the dipole contribution ( $l=1)$ of $P / P_{0}$ is null when the anapole condition is fulfilled.

As a side note, we mention that intrinsic losses in noble metals avoid the observation of anapoles. For example, for a silver particle, although the shape of the spectrum is (a)

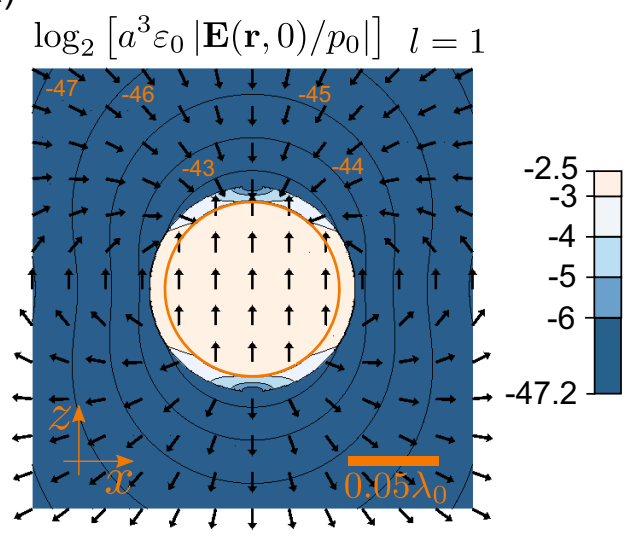

(b)

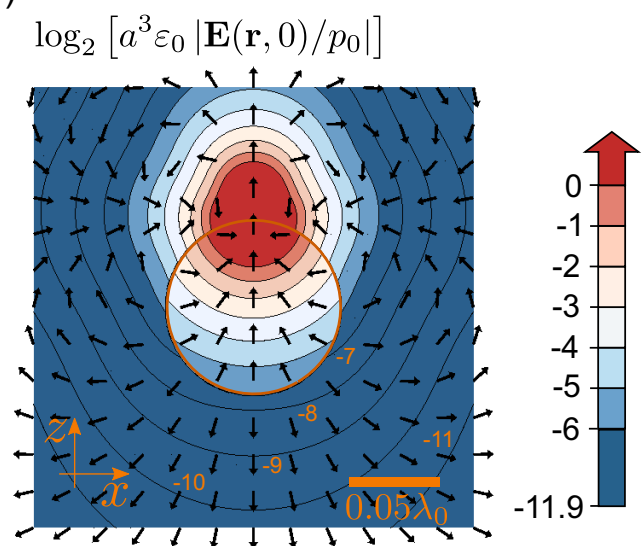

(c)
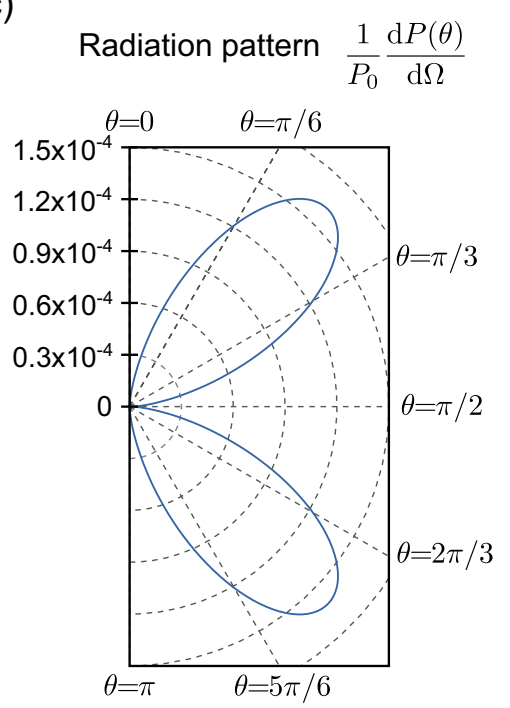

FIG. 5. Metallic sphere. Here, $k_{0} a=0.3$ and $k_{0}\left(z_{0}-a\right)=$ 0.05. (a) Contour plot of the dipolar contribution $(l=1)$ of $\left.\log _{2}\left[a^{3} \varepsilon_{0} \mid \mathbf{E}(\mathbf{r}, 0)\right] / p_{0} \mid\right]$ in the $x z$ plane. (b) Contour plot of $\log _{2}\left[a^{3} \varepsilon_{0}\left|\mathbf{E}(\mathbf{r}, 0) / p_{0}\right|\right]$ in the $x z$ plane. (c) Radiation pattern $(d P / d \Omega) / P_{0}$ vs $\theta$.

roughly similar to that of Fig. 6 , the radiated power reaches $P / P_{0}=0.616$ at anapole condition $\left[k_{0}=0.01914 \mathrm{~nm}^{-1}, a=\right.$ $15.67 \mathrm{~nm} \quad\left(k_{0} a=0.3\right), \quad z_{0}=18.29 \mathrm{~nm} \quad\left(k_{0} z_{0}=0.35\right)$, and $\epsilon_{2}=-0.329+i 0.654$ [37]]. The development of low-loss 


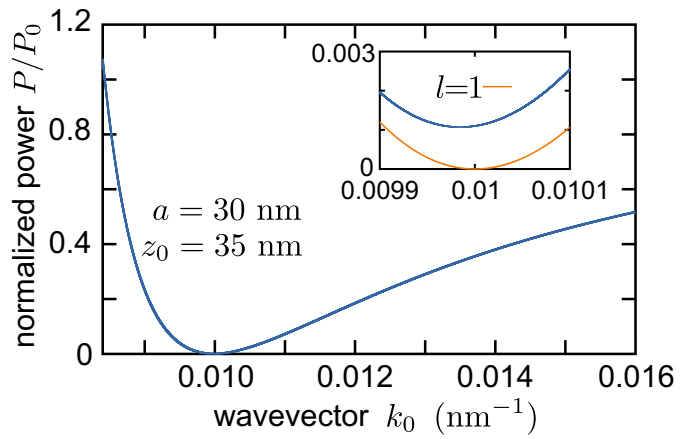

FIG. 6. Normalized power $P / P_{0}$ vs the free-space wave vector $k_{0}$ for $a=30 \mathrm{~nm}$ and $z_{0}=35 \mathrm{~nm}$. The inset shows an enlargement around the the frequency where the anapole happens, including the dipole contribution $(l=1)$ of $P / P_{0}$.

plasmonic metamaterials [38] could be used for an experimental implementation of a metallic anapole.

\section{TWO DIPOLE EXCITATION}

The residual radiated power arising from the quadrupole contribution can be further eliminated if another electric dipole with moment $\mathbf{p}=p_{0} \mathbf{n}_{z}$ is added at $\mathbf{r}_{1}=-z_{0} \mathbf{n}_{z}$. The scattering electric field $\mathbf{E}_{1}(\mathbf{r})$ produced by the dual dipole excitation is obtained by modifying (2) with the incorporation

(a)

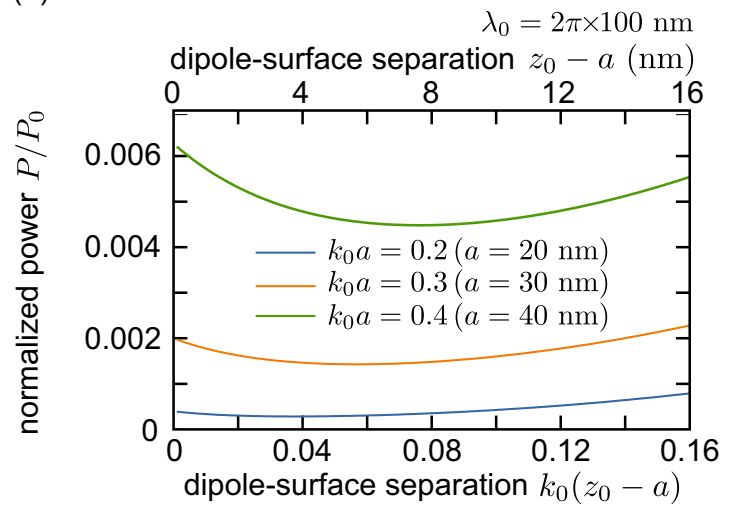

$$
\text { (b) } \quad \lambda_{0}=2 \pi \times 100 \mathrm{~nm}
$$

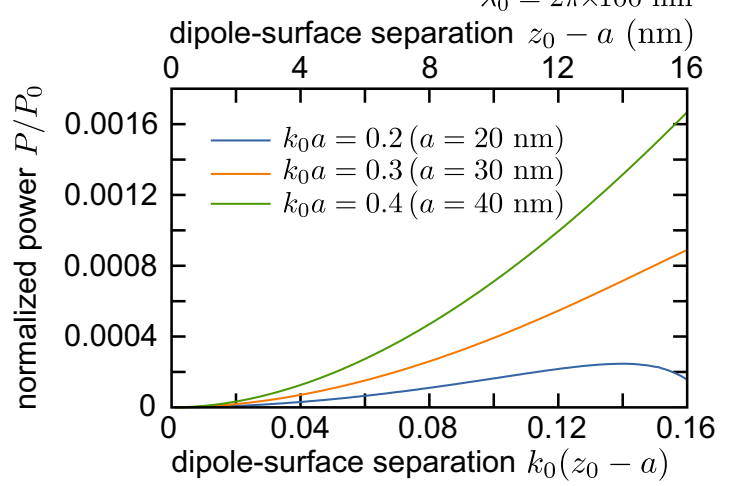

FIG. 7. Dual-dipole excitation. The normalized power $P / P_{0}$ against dipole-surface separation $k_{0}\left(z_{0}-a\right)$ for $k_{0} a=0.2,0.3$, and 0.4. (a) Dielectric sphere. (b) Metallic sphere. Conversion to nanometers is included for an anapole occurring at a free-space wavelength $\lambda_{0}=2 \pi \times 100 \mathrm{~nm}$. (a)
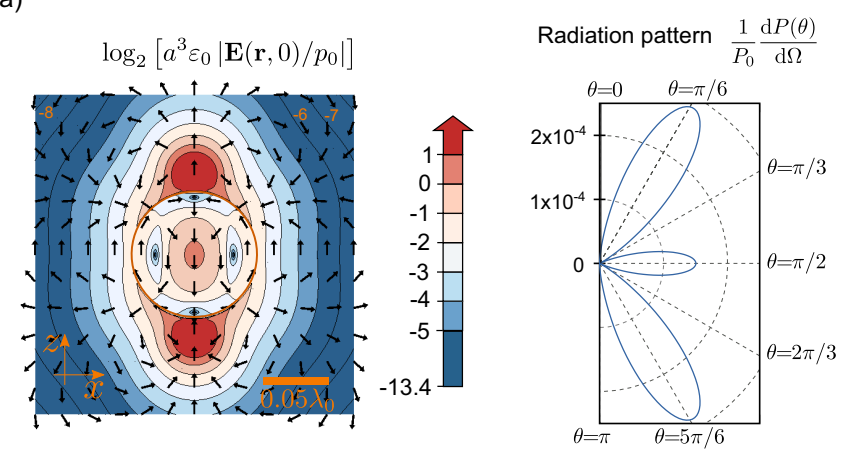

(b)
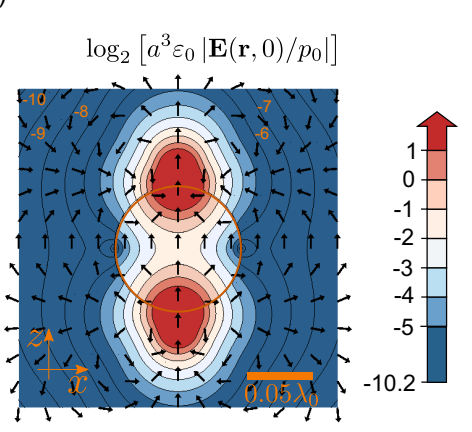

FIG. 8. Here, $k_{0} a=0.3$ and $k_{0}\left(z_{0}-a\right)=0.05$. Left panel: Contour plot of $\log _{2}\left[a^{3} \varepsilon_{0}\left|\mathbf{E}(\mathbf{r}, 0) / p_{0}\right|\right]$ in the $x z$ plane. Right panel: Radiation pattern $(d P / d \Omega) / P_{0}$ vs $\theta$. (a) Dielectric sphere. (b) Metallic sphere.

of the multiplicative factor

$$
g_{l}=1-(-1)^{l}
$$

the same factor must be used in Eqs. (6) and (7) for the direct field $\mathbf{E}_{0}(\mathbf{r})$ and internal field $\mathbf{E}_{2}(\mathbf{r})$, respectively. Hence, the effect of the additional dipole is to get rid of even multipolar orders. Concerning radiative quantities, the radiation pattern follows by inserting the factor $g_{l} g_{l^{\prime}}$ in Eq. (16), whereas the factor $g_{l}^{2}$ must be introduced in Eq. (17) for calculating the radiated power.

Figure 7 shows the normalized power $P / P_{0}$ against $k_{0}\left(z_{0}-\right.$ a) for the two dipole excitation setup; the curves consider both cases of metallic and dielectric spheres, and several particle sizes. Consequently, when the anapole condition is fulfilled for $l=1$, the dipole and quadrupole contributions to the radiated power are null. Thus, the residual radiated power is due to the octupole $(l=3)$ contribution. As expected, relative to the single dipole excitation, the normalized power is even smaller. For the dielectric sphere, the normalized power $P / P_{0}$ has been reduced by a factor in the range 20-127; the smaller the dipole surface and particle size are, the greater the reducing factor is [compare Figs. 7(a) and 1(b)], while the reduction of $P / P_{0}$ is more modest (5-25 times) for a metallic particle, as can be seen by comparing Figs. 7(b) and 4(b). Therefore, the dual-dipole excitation generates a practically ideal anapole.

Again, we consider that the particle size is $k_{0} a=0.3$ and, for both dipoles, the dipole-surface distance is $k_{0}\left(z_{0}-\right.$ $a)=0.05$. For the dielectric (metallic) sphere, the radiated power is $P / P_{0}=1.43 \times 10^{-3}\left(P / P_{0}=1.08 \times 10^{-4}\right)$, which is 50 (10) times smaller than the case of a single dipole excitation. Figure 8 illustrates the contour plot of $|\mathbf{E}(\mathbf{r}, 0)|$ 
in the $x z$ plane for the dielectric and metallic spheres when the anapole condition is fulfilled. The electric distributions of the pure anapole in the presence of two dipoles for the case of the dielectric and metallic spheres are the same as the corresponding electric-field distributions of Figs. 1(a) and 4(a) with an enhancement factor of 2, respectively. In addition, the aforementioned contour plots of $|\mathbf{E}(\mathbf{r}, 0)|$ of Fig. 8 are accompanied by their corresponding radiation patterns. By comparing Figs. 8(a) and 1(c) (dielectric sphere), as expected, the strength of the lobes of $d P(\theta) / d \Omega$ for the dual-dipole excitation is smaller than that for the single dipole excitation; the same applies for the metallic sphere, but now Figs. 8(b) and 4(c) are compared. The shapes of the radiation patterns of Fig. 8 are identical for both dielectric and metallic particles, and they come from the octupolar contribution $(l=3)$.

\section{CONCLUSIONS}

We have shown that a dipole in the presence of a subwavelength sphere can give rise to an anapole. Since the dominant radiative contribution for subwavelength particles is the lowest multipolar order (dipole), we have established the conditions for which this contribution vanishes. One of the conditions is that the exciting dipole is oriented in the radial direction and the other comes from the destructive interference between the dipole induced in the particle and the dipole moment of the exciting source relative to center of the sphere. We have found that the anapole condition can be fulfilled with either a dielectric particle having a high refractive index or a metallic particle $\left(\epsilon_{2}<0\right)$. For subwavelength spheres and surface-dipole distances $k_{0}\left(z_{0}-a\right) \ll 1$, the radiated power arising from the remaining high-order multipoles (mainly the quadrupole) is much smaller than the radiated power by the exciting dipole in the absence of the scatterer; the normalized power $P / P_{0}$ is smaller for a metallic sphere than for a dielectric sphere. We have shown that the residual power arising from high-order modes can be reduced even more by placing two electric dipoles on the opposite sides of the sphere. In this case, the even multipoles are eliminated, thus the unwanted radiated power arises from the octupolar order.

This paper might have implications for controlling radiative properties of emitters, as well as for molecular sensing and localization.
[1] P. Anger, P. Bharadwaj, and L. Novotny, Enhancement and Quenching of Single-Molecule Fluorescence, Phys. Rev. Lett. 96, 113002 (2006).

[2] Z. Ruan and S. Fan, Superscattering of Light from Subwavelength Structures, Phys. Rev. Lett. 105, 013901 (2010).

[3] I. Staude, A. E. Miroshnichenko, M. Decker, N. T. Fofang, S. Liu, E. Gonzales, J. Dominguez, T. S. Luk, D. N. Neshev, I. Brener, and Y. Kivshar, Tailoring directional scattering through magnetic and electric resonances in subwavelength silicon nanodisks, ACS Nano 7, 7824 (2013).

[4] B. Rolly, J.-M. Greffrin, R. Abdeddaim, B. Stout, and N. Bonod, Controllable emission of a dipolar source coupled with a magneto-dielectric resonant subwavelength scatterer, Sci. Rep. 3, 3063 (2013).

[5] A. F. Cihan, A. G. Curto, S. Raza, P. G. Kik, and M. L. Brongersma, Silicon Mie resonators for highly directional light emission from monolayer $\mathrm{MoS}_{2}$, Nat. Photonics 12, 284 (2018).

[6] A. Bag, M. Neugebauer, P. Woźniak, G. Leuchs, and P. Banzer, Transverse Kerker Scattering for Angstrom Localization of Nanoparticles, Phys. Rev. Lett. 121, 193902 (2018).

[7] A. Alù and N. Engheta, Achieving transparency with plasmonic and metamaterial coatings, Phys. Rev. E 72, 016623 (2005).

[8] A. Alù and N. Engheta, Multifrequency Optical Invisibility Cloak with Layered Plasmonic Shells, Phys. Rev. Lett. 100, 113901 (2008).

[9] G. N. Afanasiev and Y. P. Stepanovsky, The electromagnetic field of elementary time-dependent toroidal sources, J. Phys. A 28, 4565 (1995).

[10] E. A. Gurvitz, K. S. Ladutenko, P. A. Dergachev, A. B. Evlyukhin, A. E. Miroshnichenko, and A. S. Shalin, The highorder toroidal moments and anapole states in all-dielectric photonics, Laser Photonics Rev. 13, 1800266 (2019).

[11] N. A. Nemkov, A. A. Basharin, and V. A. Fedotov, Electromagnetic sources beyond common multipoles, Phys. Rev. A 98, 023858 (2018).
[12] B. Edwards, A. Alù, M. G. Silveirinha, and N. Engheta, Experimental Verification of Plasmonic Cloaking at Microwave Frequencies with Metamaterials, Phys. Rev. Lett. 103, 153901 (2009).

[13] A. Alù and N. Engheta, Cloaked Near-Field Scanning Optical Microscope Tip for Noninvasive Near-Field Imaging, Phys. Rev. Lett. 105, 263906 (2010).

[14] F. Bilotti, S. Tricarico, F. Pierini, and L. Vegni, Cloaking apertureless near-field scanning optical microscopy tips, Opt. Lett. 36, 211 (2011).

[15] V. A. Fedotov, A. V. Rogacheva, V. Savinov, D. P. Tsai, and N. I. Zheludev, Resonant transparency and non-trivial non-radiating excitations in toroidal metamaterials, Sci. Rep. 3, 2967 (2013).

[16] K. V. Baryshnikova, D. A. Smirnova, B. S. Luk'yanchuk, and Y. S. Kivshar, Optical anapoles: Concepts and applications, Adv. Opt. Mater. 7, 1801350 (2019).

[17] A. E. Miroshnichenko, A. B. Evlyukhin, Y. F. Yu, R. M. Bakker, A. Chipouline, A. I. Kuznetsov, B. S. Luk'yanchuk, B. N. Chichkov, and Y. S. Kivshar, Nonradiating anapole modes in dielectric nanoparticles, Nat. Commun. 6, 8069 (2015).

[18] W. Liu, J. Zhang, B. Lei, H. Hu, and A. E. Miroshnichenko, Invisible nanowires with interfering electric and toroidal dipoles, Opt. Lett. 40, 2293 (2015).

[19] W. Liu, J. Zhang, and A. E. Miroshnichenko, Toroidal dipole-induced transparency in core-shell nanoparticles, Laser Photonics Rev. 9, 564 (2015).

[20] W. Liu, B. Lei, J. Shi, H. Hu, and A. E. Miroshnichenko, Elusive pure anapole excitation in homogenous spherical particles with radial anisotropy, J. Nanomater. 2015, 672957 (2015).

[21] B. Luk'yanchuk, R. Paniagua-Domínguez, A. I. Kuznetsov, A. E. Miroshnichenko, and Y. S. Kivshar, Suppression of scattering for small dielectric particles: anapole mode and invisibility, Phil. Trans. R. Soc. A 375, 20160069 (2017).

[22] N. A. Nemkov, I. V. Stenishchev, and A. A. Basharin, Nontrivial nonradiating all-dielectric anapole, Sci. Rep. 7, 1064 (2017). 
[23] A. K. Ospanova, G. Labate, L. Matekovits, and A. A. Basharin, Multipolar passive cloaking by nonradiating anapole excitation, Sci. Rep. 8, 12514 (2018).

[24] P. C. Wu, C. Y. Liao, V. Savinov, T. L. Chung, W. T. Chen, Y.-W. Huang, P. R. Wu, Y.-H. Chen, A.-Q. Liu, N. I. Zheludev, and D. P. Tsai, Optical anapole metamaterial, ACS Nano 12, 1920 (2018).

[25] V. A. Zenin, A. B. Evlyukhin, S. M. Novikov, Y. Yang, R. Malureanu, A. V. Lavrinenko, B. N. Chichkov, and S. I. Bozhevolnyi, Direct amplitude-phase near-field observation of higher-order anapole states, Nano Lett. 17, 7152 (2017).

[26] B. Luk'yanchuk, R. Paniagua-Domínguez, A. I. Kuznetsov, A. E. Miroshnichenko, and Y. S. Kivshar, Hybrid anapole modes of high-index dielectric nanoparticles, Phys. Rev. A 95, 063820 (2017).

[27] L. Wei, Z. Xi, N. Bhattacharya, and H. P. Urbach, Excitation of the radiationless anapole mode, Optica 3, 799 (2016).

[28] A. G. Lamprianidis and A. E. Miroshnichenko, Excitation of nonradiating magnetic anapole states with azimuthally polarized vector beams, Beilstein J. Nanotechnol. 9, 1478 (2018).

[29] J. S. Totero Gongora, A. E. Miroshnichenko, Y. S. Kivshar, and A. Fratalocchi, Anapole nanolasers for mode-locking and ultrafast pulse generation, Nat. Commun. 8, 15535 (2017).
[30] G. Grinblat, Y. Li, M. P. Nielsen, R. F. Oulton, and S. A. Maier, Enhanced third harmonic generation in single germanium nanodisks excited at the anapole mode, Nano Lett. 16, 4635 (2016).

[31] M. Timofeeva, L. Lang, F. Timpu, C. Renault, A. Bouravleuv, I. Shtrom, G. Cirlin, and R. Grange, Anapoles in free-standing III-V nanodisks enhancing second-harmonic generation, Nano Lett. 18, 3695 (2018).

[32] D. G. Baranov, R. Verre, P. Karpinski, and M. Käll, Anapoleenhanced intrinsic Raman scattering from silicon nanodisks, ACS Photon. 5, 2730 (2018).

[33] L.-W. Li, P.-S. Kooi, M.-S. Leong, and T.-S. Yeo, Electromagnetic dyadic Green's function in spherically multilayered media, IEEE T. Microw. Theory 42, 2302 (1994).

[34] C.-T. Tai, Dyadic Green Functions in Electromagnetic Theory, 2nd ed. (IEEE, Piscataway, 1994).

[35] J. A. Stratton, Electromagnetic Theory (McGraw-Hill, New York, 1941).

[36] M. Choi, S. H. Lee, Y. Kim, S. B. Kang, J. Shin, M. H. Kwak, K.-Y. Kang, Y.-H. Lee, N. Park, and B. Min, A terahertz material with unnaturally high refractive index, Nature (London) 470, 369 (2011).

[37] E. D. Palik, Handbook of Optical Constants of Solids (Academic, San Diego, 1998).

[38] A. Boltasseva and H. A. Atwater, Low-loss plasmonic metamaterials, Science 331, 290 (2011). 